\title{
Clinic Evaluation of the Use of Tissue Expanders: Report of Ten Pediatric Cases
}

\author{
Hugo Juárez Olguín ${ }^{1 *}$ (D), Gerardo Fernández Sobrino², Lulú Sánchez Reyes ${ }^{3}$, Francisca Trujillo Jiménez ${ }^{4}$ \\ and José Francisco González Zamora ${ }^{5}$
}

${ }^{1}$ Laboratory of Pharmacology, Instituto Nacional de Pediatria, Mexico

${ }^{2}$ Department of Pharmacology, Universidad Nacional Autónoma de México, Mexico

${ }^{3}$ Service of Plastic Surgery, Instituto Nacional de Pediatria, Mexico

${ }^{4}$ Laboratory of Experimental Surgery, Instituto Nacional de Pediatria, Mexico

\begin{abstract}
Purpose: The aim of the present work was to know and assess the performance and the usefulness of employing TE in pediatric population.

Methods: Ten children candidates to use TE that seek for attention at National Institute of Pediatrics in Mexico were analyzed.
\end{abstract}

Results: Ten patients, aged 3 to 16 years, who presented congenital nevi, burns and necrotizing fasciitis. Four of the

Conclusions: Because of the favorable results obtained in most of our patients, we conclude that the use of expanders is the best option for the treatment of dermatological affectations.

Keywords

Aesthetic procedures, Tissue expanders, Plastic technique, Reconstruction

\section{Introduction}

In 1957, Neumann [1], published an experiment on an atrium reconstruction case using an inflatable rubber balloon instrument which was grafted under the skin and inflated by injecting liquid through tubes that communicated with the outside. In the experiment, the tubes were made of flexible polyurethane and emerged into the outside through an incision on the skin and connected to an external valve which allows progressive injection of liquid into the implant. In this way, Neumann introduced, in the area of medicine, the use of rudimentary tissue expanders.

Radovan [2] put a touch of perfectionism in tissue expanders and published an experiment on self-designed tissue expanders for breast reconstruction; and thus, brought into manifestation the popularity of the technique in the medical field. In the experiment, Radovan implanted a balloon under the skin of a patient, which by means of small tube with an outlet in the outside could be inflated at will. This implant had, in addition, another independent tubular system that allowed liquid extraction in the event of over-distension. It was in this way that the hystic expansion device achieves medical recognition.
At the same time, Austad and Rose [3], self-inflated silicone expander whose principle lies in the placement of hyperosmolar substances inside a semi-permeable membrane; and thus, created an osmolarity gradient that favors gradual volume increase of the expander.

In recent years, the number of pediatric patients seeking for attention at National Institute of Pediatrics (INP in its Spanish acronym) due to dermatological problems has increased. Most of these problems require surgical procedures for their treatment and their management has revolutionized due to the application of tissue expansion technique. However, the

*Corresponding author: Hugo Juárez Olguín, Department of Pharmacology, Laboratorio de Farmacología, Instituto Nacional de Pediatría, Avenida Imán $\mathrm{N}^{\circ} 1$, 3rd piso Colonia Cuicuilco CP 04530, Mexico City, Mexico, Tel/Fax: 5255-56161489

Accepted: October 19, 2020

Published online: October 21, 2020

Citation: Olguín HJ, Sobrino GF, Reyes LS, et al. (2020) Clinic Evaluation of the Use of Tissue Expanders: Report of Ten Pediatric Cases. Arch of Pedia Surg 4(2):66-71 
precise usefulness of this technique as well as the exact characteristics and indications to perform this procedure are not known; thus, making it difficult to bring this novel process to practice [4].

Tissue expansion is useful in the field of surgery since the application of this technique has succeeded in reconstructing or improving different dermatological aspects in pediatric patients, giving them both the desired physical and mental remedies [5].

Derived from the above observations and findings, the aim of the present work was to know and assess the performance and the usefulness of employing TE in the pediatric population that seek for attention at INP, as well as to know the therapeutic indications and the results obtained in the applications of the technique.

\section{Material and Methods}

This study was conducted by the Pharmacology laboratory Department of INP from March 2011 to April 2018. It involved 10 pediatric female patients with ages ranging from 3 to 6 years attended at the hospital for presenting dermatological affectations that went from burns, giant melanocytic nevi (GMN) to necrotizing fasciitis (NF). Hence, all the patients were candidates for the placement of tissue expanders (TE).

An expander was placed on the 10 patients, and was weekly filled and constantly monitored until maximum expansion was achieved. 4 of the 10 TE placed achieved an expansion of $100 \%$ of their capacity. The expander was left for an average duration of 60 days (two months) until most of them presented infection or naturally rejected by the body. After the withdrawal of the expanders, a careful follow-up of the patients was implemented. The result was fantastically favorable in most of them.

\section{Results}

This work describes the principal clinical findings after the placement of TE in 10 patients, aged 3 to 16 years for an average of 6 months. The patients presented congenital nevi, burns and NF. 4 of the expanders achieved $100 \%$ of expansion. Most of them should have been removed due, principally, to contamination problem.

Of a total of 10 female patients, five $(n=5)$ had GMN; two $(n=2)$ presented NF; two $(n=2)$ with BS; and one $(n=1)$ presented non-neoplastic nevus (NNN) (Table 1). The age range of the patient was between 3 and 16-years-old (average 10 years). A higher prevalence of GMN occurred in patients under 10 years.

Although the dermatological affectation zones were variable, we were able to discern a recurrence in the lumbar re-

Table 1: Medical indication for the application of the tissue expander.

\begin{tabular}{|l|l|}
\hline Diagnosis & Number of Cases \\
\hline Giant melanocytic nevi (GMN) & 5 \\
\hline Necrotizing fasciitis (NF) & 2 \\
\hline Burn scars (BS) & 2 \\
\hline Non-neoplastic nevus (NNN) & 1 \\
\hline
\end{tabular}

Table 2: Dermatological affectation zones considered for the indication.

\begin{tabular}{|l|l|l|}
\hline No. of Patient & Diagnosis & Affected Zones \\
\hline 1 & Burn scars & Right forearm \\
\hline 2 & Necrotizing fasciitis & Right hemifacial \\
\hline 3 & Burns scars & Right hemithorax \\
\hline 4 & Giant melanocytic nevi & Abdominal perineum \\
\hline 5 & Giant melanocytic nevi & Lower back \\
\hline 6 & Giant melanocytic nevi & Right gluteus \\
\hline 7 & Giant melanocytic nevi & Thigh \\
\hline 8 & Giant melanocytic nevi & Lumbar region \\
\hline 9 & Non-neoplastic nevus & $\begin{array}{l}\text { From lumbar region } \\
\text { until right knee }\end{array}$ \\
\hline 10 & Necrotizing fasciitis & $\begin{array}{l}\text { Lumbar region and } \\
\text { right muscle }\end{array}$ \\
\hline
\end{tabular}

\section{Days of use per patient}

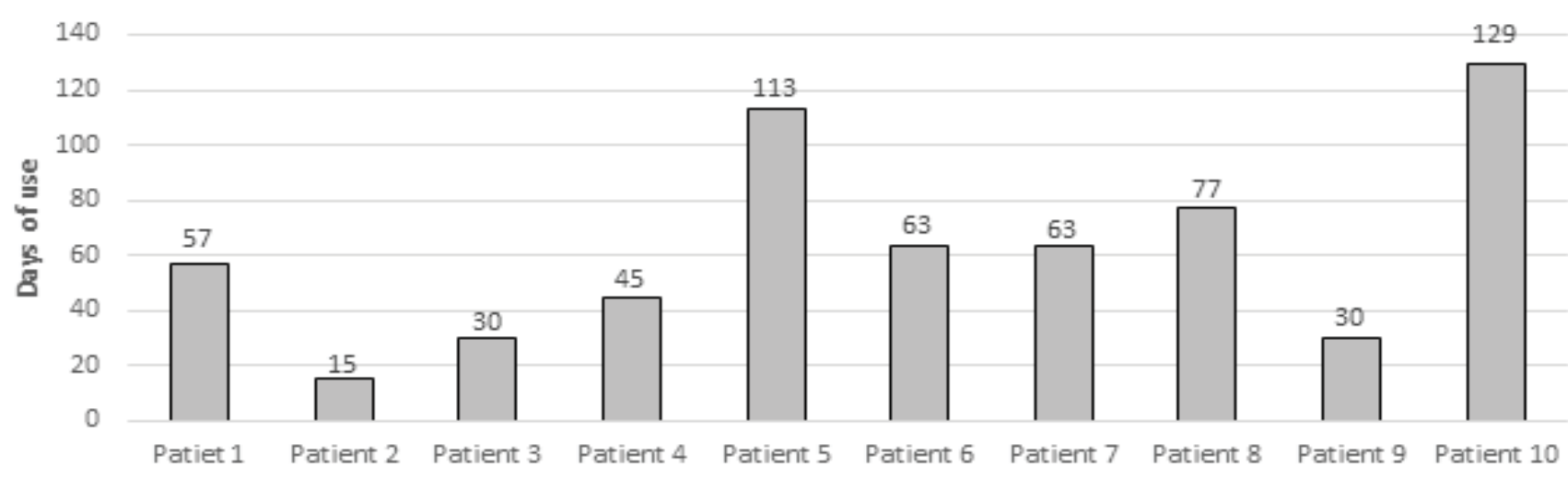

Patients

Figure 1: Days of use tissue expanders per patient. 


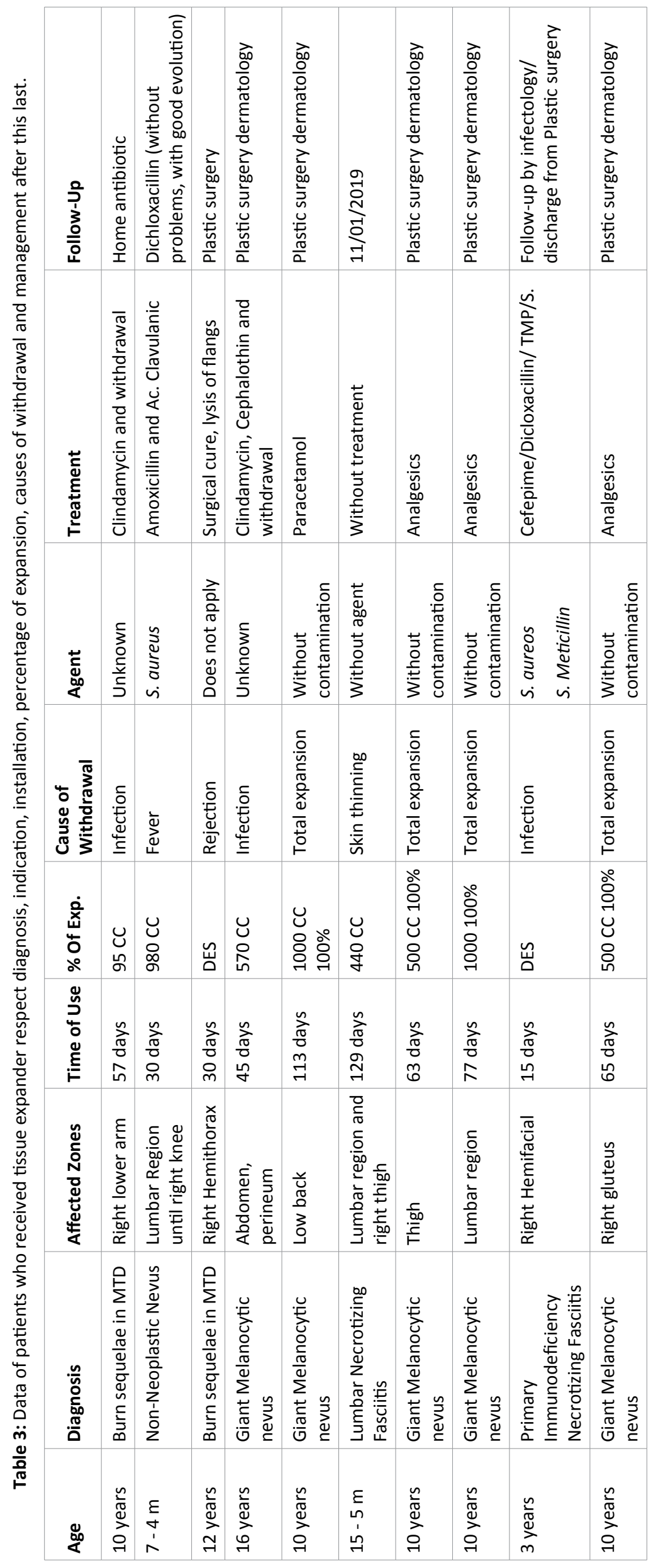


gion in 3 cases (Table 2). TE average duration was 2 months (60 days). The exact days of use is illustrated in (Figure 1).

When the expansion is observed, 4 of the expanders reached their maximum expansion (100\%) and were withdrawn. The rest of the 6 expanders did not achieve to reach their maximum due to infection. 1 was rejected by the patient's body and was removed for thinning down the patient's skin thickness (Table 3).

Finally, regarding the follow-up of the 10 patients (100\%), $7(70 \%)$ finished the plastic surgery, $1(10 \%)$ was with antibiotic treatment from home and another 1 (10\%) with doxycycline, all with favorable results. The remaining one is presently under follow-up (Figure 2).

\section{Discussion}

Melanocytic nevi are frequently observed in pediatric population. The classifications general fall into two broad groups: The congenital melanocytic nevi (CMN), generally present at birth or in childhood; and the acquired melanocytic nevi (AMN). The CMN are sub-classified according to size and morphological characteristics. Neurocutaneous melanosis and melanoma represent two important complications of this group with a general risk determined by the size of the nevus, its location, appearance and the number of satellite lesions. The AMN tends to appear in childhood and increase in number during adolescence. Risk factor for melanoma in children includes family history of melanoma, excessive exposition to ultraviolent light, lightly pigmented skin and immunosuppression.
With the surgical approach, the observations in clinical test and other studies can be confirmed. Tissue expanders offer unimaginable benefits and has been considered the most viable option for the management of GMN. This view is buttressed by many studies remarking this treatment approach as the most feasible and practicable option for the treatment $\mathrm{GMN}$, as the cases reported in this study.

In terms of necrotizing fasciitis (NF), some authors described it as a highly destructive progressive infection of the soft tissues that produces skin, subcutaneous fat and fascia necrosis [6]. However, notwithstanding the increase in the cases of NF in the last years, it continues to be infrequent ailment. The incidence in children has a toll of 0.08 in every 100,000 inhabitants $[7,8]$.while the estimated mortality is $73 \%$ in severe forms of the cases[6].

Nevertheless, this figure can undergo variations depending on the age ( $18 \%$ in children), [8] and comorbidity conditions (diabetes, chronic renal insufficiency, etc) [9]. There are many predisposing conditions for NF. Undoubtedly, any clinical entity with immunosuppression constitutes the best scenario to develop the disease [8].

This factor was observed in our patient, who in addition to having NF, also had primary immunodeficiency. Other predisposing conditions in children are post-operative wound, trauma, diabetes mellitus, lacerations, insect bites and others such as malnutrition and chickenpox. This later condition represents an optimal terrain to trigger the disease. The infection affects any site of the organism. In a study published by Fustes [8], in 39 children, it was found that there is no predispo-

\section{Patients' follow-up}

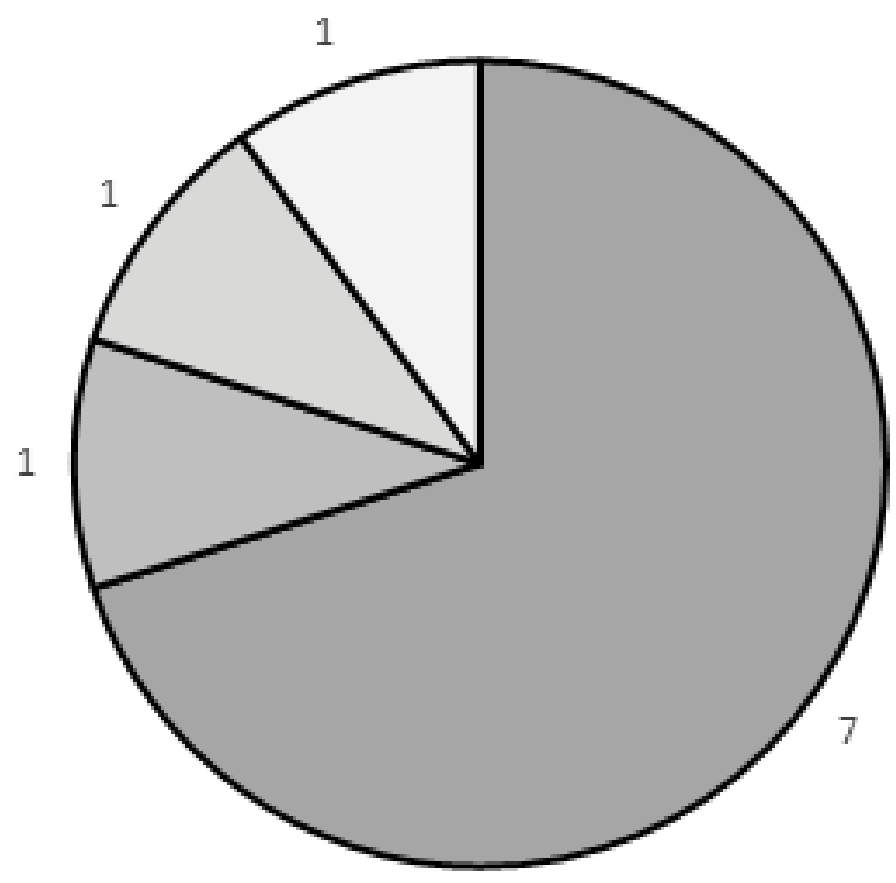

Plastic Surgery

Antibiotics at home

Doxycycline tr eatment

口 under follow-up

Figure 2: Follow-up of the patients after management with their tissue expander. 
sition by gender and that $77 \%$ of the lesions are unique while the extremities are the most affected (54\%) with the most common pre-existing conditions being malnutrition (36\%) and chickenpox (33\%). The disease location is closely associated with the mortality. Undoubtedly, the lesions in the scalp, face and trunk are often of greater severity [8].

In the present study, one of the two patients with NF presented lesions in the lumbar region, thus, it was not something so complicated to treat; however, the other patients presented lesions in the right hemifacial and accordingly, as the author mentioned, it was a case of a disease with high mortality because of its location and hence, more serious.

In the initial stages, the skin presents discolored plaques with undefined edges, edema and signs of lymphangitis. The patients manifest significant pain, usually disproportional with the size of the lesion. In this stage, it is easy to confuse the lesion with cellulitis. In acute cases, two to three days after, the infection progresses by forming an erythematous plaque that later turns purple and blackish with bag-like areas on the surface marking the point at which a truly devastating necrotic process begins.

The most effective measures to reduce the mortality include early diagnosis of the disease, antimicrobial therapy and surgical debridement. Many of the patients require intensive therapy assistance because of hemodynamic problems and multi organ failure. As already mentioned, the surgical treatment is the basis of NF management and its delay is the main determinant factor of mortality. The objectives of surgery are to confirm diagnosis of suspicion, perform necrosectomy, an extensive surgical debridement and drainage of the possible existing collections, and to obtain samples for histological and microbial diagnosis. It is recommended to perform surgical re-evaluation of the condition, at least, 24 hours after the initial procedure, and later, as many times as necessary until all necrotic tissues are eliminated and local infection control is achieved [10].

Due to the above, the decision to place tissue expanders in our patients was taken, in order to obtain a better future prognosis and to succeed in their recovery of health as best as possible. After the withdrawal of the TE, one of them succeed in undergoing surgery and the other patient continues his evolution, but in both, the decision taken was the placement of expander.

Burns are common, especially in children who are usually frequent victims of hot water spillage. The most severe lesions, but fortunately very rare, are the events of thermal burns in closed places (burns in house fire, car fire, etc.) or serious electrical burns [11]. The objective of the initial treatment is to achieve the fastest and most reliable closure as possible. In the absence of bone exposition, the best primary treatment continues to be thin skin graft. Apart from its simplicity and speed, the main advantage is that it does not produce deep scars [11]. The surgical technique comprises of two phases. The first phase is the placement of one or more prosthesis and the second phase, usually done three months after, consists of the extraction of the prosthesis and the production of expanded flaps [12].
Regarding chest burns, such as that presented in one of our patients, breast deformity is always involved. Breast deformity secondary to burns includes abnormal scars, small contraction bands, contour deformity or distorted scars, hypoplasia or absence of breast and the abnormalities of the areolar-nipple complex $[13,14]$. Breast reconstruction is often multi-stage with separated procedures for the initial operation, and for the augmentation and administration of the areolar-nipple complex. Also, it can include the management of secondary deformities of scars and procedures in the contrary breast to achieve symmetry [15].

The use of TE brought a new era in the treatment of BS because they provide skin of the same color and texture, sensitivity and annexes, thus facilitating primary closure and without producing damage in the donor site. The procedure can be repeated in the same segment (re-expansion) to repair extensive sequelae and to expand the donor sites for the recollection grafts $[16,17]$. Thus, confirming that the use of expanders in the two cases of BS in our study was a good decision, since in addition to being the best technique, it is the best option for our patients, which allowed the recovery of their skin aspects in their totality.

\section{Conclusion}

The experiences gathered in our patients in this study and buttressed by other studies of many authors lead to the conclusion that TE for the treatment of GMN, NF and BS are very useful, since it is the most effective way to achieve a satisfactory recovery in the patients. In addition, it is not a risky technique when performed in the correct way by health professionals. They provide to the patients the hope of almost complete recovery without many complications. In the same way, they ensure tissue reconstruction without the likelihood of rejection because the grafted skin is from the same body of the patient, thus, providing a better result both in the aspects of aesthetic and immunology. Because of the favorable results obtained in most of our patients, we conclude that the use of expanders is the best option for the treatment of dermatological affectations.

\section{Conflict of Interest}

The authors declare that they have no conflict of interest.

\section{Ethical Approval}

All procedures performed in studies involving human participants were in accordance with the ethical standards of the institutional and/or national research committee and with the 1964 Helsinki declaration and its later amendments or comparable ethical standards.

\section{Funding}

No funding was obtained from external sources for this study.

\section{Acknowledgements}

We thank Dr. Cyril Ndid i Nwoye Nnamezie, an expert translator whose native language is English, for his help in preparing this manuscript. 
Besides, we are very grateful to National Institute of Pediatrics, Mexico City for the provision of Federal Resources to support this work.

\section{References}

1. Neumann CG (1946) The expansion of an area of skin by progressive distention of a subcutaneous balloon; use of the method for securing skin for subtotal reconstruction of the ear. Plast Reconstr Surg 19: 124-130.

2. Radovan C (1984) Tissue expansion in soft tissue reconstruction. Plast Reconstr Surg 74: 482-492.

3. Austad ED, Rose GL (1982) A self-inflating tissue expander. Plast Reconstr Surg 70: 588-594.

4. Bjornson LA, Bucevska M, Verchere C (2019) Tissue expansion in pediatric patients: A 10-year review. J Pediatr Surg 54: 14711476.

5. LoGiudice J, Gosain AK (2003) Pediatric tissue expansion: Indications and complications. J Craniofac Surg 14: 866-872.

6. Kotrappa KS, Bansal RS, Amin NM (1996) Necrotizing fasciitis. Am Fam Physician 53: 1691-1697.

7. File TM, Tan JS, Dipersio JR (1998) Group A streptococcal necrotizing fasciitis. Diagnosing and treating the "flesh eating bacteria syndrome". Clev Clin J Med 65: 241-249.

8. Fustes-Morales A, Gutierrez Castrellon P, Durán-McKinster C, et al. (2002) Necrotizing fasciitis: Report of 39 pediatric cases. Arch Dermatol 138: 893-899.
9. Hoeffel JC, Hoeffel F (2002) Necrotizing fasciitis and purpura fulminans. Plast Recon Surg 109: 2165.

10. Sudarsky LA, Laschinger JC, Coppa GF, et al. (1987) Improved results from a standardized approach in treating patients with necrotizing fasciitis. Ann Surg 206: 661-665.

11. Voulliaume D, Chichery A, Chekaroua K, et al. (2007) Tissue expansion in surgical treatment of burn scars of the scalp. Ann Chir Plast Esthet 52: 590-599.

12. Foyatier JL, Comparin JP, Latarjet J, et al. (1993) Forum on tissue expansion. Repair of sequelae of facial burns by cervical cutaneous expansion. Ann Chir Plast Esthet 38: 27-33.

13. MacLennan SE, Wells MD, Neale HW (2000) Reconstruction of the burned breast. Clin Plast Surg 27: 113-119.

14. Fish J (2006) Reconstruction of the burned breast and abdomen. In: Sood R, Achauer BM, Achauer and Sood's burn surgery: Reconstruction and rehabilitation. Saunders Elsevier, Philadelphia, 264-272.

15. Wainwright DJ (2009) Burn reconstruction: The problems, the techniques, and the applications. Clin Plast Surg 36: 687-700.

16. Spence RJ (1992) Experience with novel uses of tissue expanders in burn reconstruction of the face and neck. Ann Plast Surg 28: 453-464.

17. Tavares Filho JM, Belerique M, Franco D, et al. (2007) Tissue expansion in burn sequelae repair. Burns 33: 246-251. 